\title{
tic\&société
}

Vol. 6, $\mathrm{N}^{\circ} 1$ | Second semestre 2012

Mutations du journalisme : nouveaux dispositifs, nouvelles pratiques

\section{Le dispositif télévisuel de la version québécoise de Tout le monde en parle}

Espace déictique, identité discursive et cadre participatif

\section{Pierre Barrette}

\section{OpenEdition \\ Journals}

\section{Édition électronique}

URL : http://journals.openedition.org/ticetsociete/1237

DOI : 10.4000/ticetsociete. 1237

Éditeur

Association ARTIC

\section{Référence électronique}

Pierre Barrette, "Le dispositif télévisuel de la version québécoise de Tout le monde en parle»,

tic\&société [En ligne], Vol. 6, N¹ I Second semestre 2012, mis en ligne le 30 novembre 2012, consulté le 02 mai 2019. URL : http://journals.openedition.org/ticetsociete/1237 ; DOI : 10.4000/ ticetsociete. 1237 


\title{
Le dispositif télévisuel de la version québécoise de Tout le monde en parle: espace déictique, identité discursive et cadre participatif
}

\author{
Pierre BARRETTE \\ École des Médias (UQAM) \\ UQAM \\ Case postale 8888 , succ. Centre-ville $\square$ Montréal \\ (Québec) H3C 3P8 \\ Canada
}

\section{barrette.pierre@uqam.ca}

Pierre Barrette est docteur en sémiologie et professeur à l'École des médias de l'UQÀM. II enseigne la télévision, le cinéma, les théories de la communication et publie abondamment, d'une part dans le domaine de la sémiologie et de l'analyse des médias, d'autre part à titre de collaborateur à diverses revues culturelles. De 2003 à 2008, il a participé à la préparation des Cahiers du Gerse, revue spécialisée de cinéma et de communication publiée par les Presses de I'Université du Québec. II a fait partie du comité de rédaction de la revue québécoise de cinéma 24 Images pendant 12 ans et est maintenant le responsable de la section Médias \& société de la revue en ligne Hors-champ, au sein desquelles il a fait paraître ces dernières années plus de 300 articles de critique et d'analyse orientés vers le cinéma et la télévision. Spécialiste de la télévision québécoise, il consacre aujourd'hui ses recherches à la sémiologie des genres, à l'histoire de la télé ainsi qu'aux mutations contemporaines de l'institution télévisuelle. 


\title{
Le dispositif télévisuel de la version québécoise de Tout le monde en parle : espace déictique, identité discursive et cadre participatif
}

\begin{abstract}
Résumé
Cet article se penche sur le dispositif télévisuel particulier de l'émission Tout le monde en parle, à cheval entre les affaires publiques et le domaine des variétés. Ce n'est pas tant la nature des échanges qui retiendra notre attention dans la présente analyse que le dispositif particulier à l'intérieur duquel ils prennent place, offrant un cadre énonciatif propice à générer un certains nombre d'effet largement indépendants du contenu discursif proprement dit. En accord avec notre conception de l'espace déictique, l'objectif est de donc de rendre compte, une fois accomplie l'étape proprement descriptive, d'une part de la manière dont le dispositif manifeste l'« état du champ » en construisant son identité discursive, et d'autre part du type de cadre participatif qu'il induit.
\end{abstract}

Mots-clés : Télévision, information, infodivertissement, dispositif, Tout le monde en parle (émission de télévision), Québec

\begin{abstract}
This article focuses on the specificities of the popular Quebec television show Tout le monde en parle, which is half way between public affairs and variety. It is not so much the nature of the verbal exchanges that hold our attention in this analysis, as the particular apparatus within which they take place, providing a framework conducive to generate a certain number of effects, largely independent of the discursive context. After a thorough description of the show's format and operations, In keeping with our conception of space deixis, our objective is to establish, firstly how the device shows the "state of the field" by building its discursive identity, and secondly, the type of participation framework that it induces.
\end{abstract}

Keywords: television, information, infotainment, "Tout le monde en parle" (TV show), Quebec 
Le dispositif télévisuel de la version québécoise de Tout le monde en parle :

espace déictique, identité discursive et cadre participatif

\section{Resumen}

Este artículo analiza el dispositivo televisivo "Tout le monde en parle", programa de la televisión francófona de Canadá, que se sitúa a caballo entre el ámbito de los asuntos públicos y el de las variedades. El análisis no se centra tanto en la naturaleza de los intercambios que se dan en este programa, sino más bien en el dispositivo dentro del cual dichos intercambios tienen lugar; ofrece además un marco enunciativo propicio para generar un cierto número de efectos, en gran medida, independientes del contenido discursivo. Una vez cumplida la etapa propiamente descriptiva y de acuerdo con nuestra concepción del espacio deíctico, el objetivo será doble: por un lado, analizar la manera en que el dispositivo manifiesta el 'estado del campo', construyendo su identidad discursiva, y por otro lado, el tipo de marco participativo que induce.

Palabras claves: televisión, información, info-entretenimiento, dispositivo, "Tout le monde en parle" (programa de televisión), Quebec. 
Parmi les mutations de fond qui affectent le champ journalistique depuis quelques années, la montée en force de ce que d'aucuns appellent l'infotainment ou infodivertissement (Bastien, 2007 ; Brants, 2003 ; Thussu, 2007) constitue sinon une menace, du moins un défi important lancé à la manière dont on traite traditionnellement de l'actualité dans les médias, et en particulier des questions politiques. Ce qui est mis sur la table dans ces nouvelles configurations de la parole politique marquées notamment par la désacralisation, l'informalisation et l'assouplissement des échanges et des rituels (Neveu, 2007), ce sont bien entendu les « conditions sociopolitiques du débat public " (Le Foulgoc, 2007). Celles-ci tendraient à donner une place de plus en plus congrue aux questions de fond cependant que la vie privée des individus serait mise de l'avant et exploitée (un phénomène surtout présent en France, il faut le dire, et généralement désigné par l'expression peoplelisation), parfois avec l'assentiment des principaux intéressés qui y trouvent une belle occasion d'utiliser les médias à des fins de relations publiques. L'exemple désormais canonique d'un Jacques Chirac en tournée aux Pays-Bas et qui refuse toute entrevue à l'exception d'un passage sur le plateau d'une émission de variétés rappelle par ailleurs nombre de cas semblables plus près de nous, notamment celui de la ministre québécoise Christine St-Pierre qui, à l'automne 2011, décidait d'annuler ses engagements auprès du Téléjournal de la Société Radio-Canada (la télévision publique francophone canadienne) dans le but de répondre à une invitation du talk-Show Tout le monde en parle (aussi diffusé par Radio-Canada) qui réclamait l'exclusivité.

C'est cette situation très particulière de Tout le monde en parle (TLMEP dans le reste de l'article), à cheval entre les affaires publiques et le domaine des variétés, qui nous intéressera dans la suite du présent article. Notre hypothèse est que la dimension proprement politique de TLMEP ne dépend pas principalement de l'origine des invités sur le plateau de l'émission; elle relèverait en bonne partie de son dispositif technique et énonciatif particulier qui, par-delà la nature des échanges discursifs, contribue à faire du plateau de l'émission un simulacre de l'espace public. 
Le dispositif télévisuel de la version québécoise de Tout le monde en parle : espace déictique, identité discursive et cadre participatif

\section{TLMEP et l'infodivertissement}

Reprise d'un format français créé et animé par Thierry Ardisson sur France 2 (de 1998 à 2006), la version québécoise animée par Guy A. Lepage (depuis 2004) partage avec le modèle français son caractère " d'arène moderne et "branchée" qui donne une visibilité médiatique à des personnalités connues faisant l'actualité artistique et politique du moment "(Amey, 2009, p.196). Le " cas » de l'émission dominicale québécoise est toutefois assez singulier : sa longévité (l'émission en est à sa 7e saison en 2011-2012) et sa popularité (TLMEP est pratiquement le seul succès de la SRC à se classer régulièrement parmi les cinq émissions les plus regardées, selon les sondages BBM) nous forcent à y voir un rendez-vous hebdomadaire incontournable, possiblement l'un des rares au Québec en cette ère de télévision hyperspécialisée, une télévision qui, selon Jean-Louis Missika, "explose en bouquets de programmes, se fragmente en chaînes ultra-thématiques, se désarticule en vidéo à la demande, se package en service push sur le mobile, se télécharge sur Internet, se podcaste sur l'iPod, s'individualise en blog et vlog » (Missika, 2006, 4e de couverture). Le fait que l'émission se retrouve régulièrement au centre de polémiques, dont plusieurs concernent la frontière entre le journalisme et les variétés, permet également d'y voir un laboratoire privilégié pour l'analyse de l'infodivertissement et de la peoplelisation, les deux phénomènes évoqués cidessus formant la face la plus visible des mutations qui affectent le journalisme contemporain.

TLMEP est caractéristique à plus d'un égard de ces mutations, qu'on pourrait résumer avec Nicolas Pelissier et Daniel Bougnoux autour de trois grands axes généraux : la déprofessionnalisation de l'identité journalistique, liée notamment au développement des nouvelles pratiques sur le web et d'un journalisme dit " citoyen » ; la transformation du contexte économique et institutionnel de la pratique, marquée en outre par les phénomènes de concentration et de convergence ; enfin, le morcellement, la spécialisation et la fragmentation des publics, qui adoptent de plus en plus face à l'offre journalistique une attitude de "clients ". II nous semble que le dispositif propre de TLMEP, avec ses animateurs-humoristes qui adoptent une posture habituellement réservée aux journalistes professionnels et présentent dans un cadre "divertissant " des contenus traités ailleurs par les médias d'information traditionnels, constitue une illustration manifeste de ce nouveau contexte.

La quasi-totalité des études consacrées à TLMEP situe en effet l'émission au cœur de la problématique de l'infotainement et de ce que d'aucuns ont qualifié 
de " dérive de l'espace public à l'ère du divertissement » (Barrette, Paquette et Perraton, 2007). Pourtant, le phénomène n'est pas nouveau; si on en croit Frédérick Bastien, qui propose dans sa thèse une histoire de l'infodivertissement à la télévision québécoise (2007, p. 107 et suivantes), déjà dans les années 1950, certaines émissions d'affaires publiques de Radio-Canada proposaient un mélange d'information à contenu politique et de divertissement. Mais il reste clair toutefois que le contexte des deux dernières décennies, caractérisé par une compétition renforcée entre les chaînes et une tendance accrue à l'hybridité générique, a grandement favorisé son développement. L'infodivertissement prend un caractère quelque peu différent dépendant des contextes où il est étudié; souvent associé en Europe à une américanisation des pratiques journalistiques liée à l'instrumentalisation commerciale croissante de l'information, il semble en effet faire partie du système de l'information aux États-Unis où il représente une stratégie sinon généralisée, à tout le moins fortement intégrée à la culture journalistique. La situation canadienne et tout particulièrement québécoise, avec son régime de télévision mixte (public et privé), se laisse bien décrire comme un cas d'hybridité (Bastien, 2007).

Parmi les analyses suffisamment élaborées qu'ont suscitées les versions française et québécoise de TLMEP (Neveu, 2003; Amey, 2007 ; Rochette, 2010 et Blouin, 2007), on peut identifier le recours à deux axes méthodologiques distincts: le premier consiste à isoler et à traiter indépendamment des autres éléments de l'émission la dimension politique de son contenu. Émilie Rochette, par exemple, propose d'analyser les entrevues de politiciens à TLMEP « en fonction des contenus et de l'organisation des entrevues correspondant au genre médiatique infodivertissement, de manière à révéler les éléments relatifs à l'information politique et au divertissement (2010, p. 8) ». Autrement dit, il s'agit d'activer un cadre d'analyse dérivé de la sociolinguistique permettant une description fine des interactions langagières entre l'animateur et les politiciens, et ce dans le but de départager la portion du discours qui relève du domaine politique de celle qui relève de la sphère privée. Rochette arrive d'ailleurs à des conclusions qui tendent à contredire le discours « convenu » concernant TLMEP :

Les résultats tendent à démontrer que les sujets associés au divertissement sont beaucoup moins présents que ce que semble laisser croire la croyance populaire. L'étude montre également qu'en contexte d'infodivertissement, la performance du politicien est liée à sa capacité à maintenir son identité professionnelle et à s'adapter spontanément à un 
Le dispositif télévisuel de la version québécoise de Tout le monde en parle: espace déictique, identité discursive et cadre participatif

mode interactionnel plus près de son identité personnelle (2010, p. i).

II est à noter que l'auteure ne tient aucun compte dans son analyse du contexte énonciatif à l'intérieur duquel prennent place les entrevues qu'elle analyse, ce en quoi son analyse est largement complémentaire de la réflexion d'Érik Neveu, représentative pour nous du deuxième axe méthodologique. Dans un article qu'il consacre partiellement à la version française de TLMEP (deux autres émissions apparentées y sont également analysées), Neveu s'attarde en effet à questionner « la manière de mettre en scène et en question le personnel politique » (p.96), à partir notamment - le contenu des entrevues y est aussi analysé, toutefois de manière plus informelle - d'une analyse sommaire du dispositif télévisuel de l'émission. II identifie en outre les traits fédérateurs suivants, propres à la catégorie des talk-shows qui mêlent sur leur plateau invités politiques et vedettes variées (sports, showbizz, etc.): le parti-pris de décontraction qui règne sur le plateau, l'assouplissement des règles vestimentaires, l'informalisation des modes d'adresse (tutoiement vs vouvoiement), le relâchement de l'expression ainsi que la mixité sociale du statut des invités. Le travail de Neveu a le mérite de mettre de l'avant des éléments très concrets de la situation de communication qui ne relèvent pas directement du domaine discursif, mais nous croyons pour notre part qu'il reste essentiel de pousser beaucoup plus loin cette analyse si l'on veut être en mesure d'en saisir tous les impacts.

En effet, cette bipartition de la recherche correspond à la nature même du talk-show qui, comme le débat télévisé, doit être considéré du double point de vue du phénomène langagier et du phénomène audiovisuel. II est en effet régi par au moins deux dispositifs, qu'il est important de distinguer ici : un dispositif conversationnel (tel qu'analysé en détail par Rochette) et un dispositif plus proprement télévisuel. Ne serait-ce que par son titre, TLMEP se présente d'ailleurs sous ce double registre d'une communauté inclusive, mise en scène à travers un dispositif précis (Tout le monde...) fondée sur la conversation (... en parle). C'est ce double caractère, à la fois identitaire et discursif mis en place par l'émission et ses agents, qui lui permet de proposer un simulacre d'agora, un lieu qui se construit tel un espace de prise de paroles, d'échange et de confrontation et où se profile symboliquement le projet d'une unité réalisée audelà des différences. C'est aussi lui, dans une large mesure, qui nous permet de penser TLMEP dans le contexte des mutations contemporaines du journalisme, et en particulier de la dépendance croissante dans laquelle se trouvent les médias et les journalistes face à leurs publics. 
Toutefois, et même si cette dimension est bien entendu centrale, ce n'est pas tant la nature des échanges qui retiendra notre attention dans la présente analyse que le dispositif particulier à l'intérieur duquel ils prennent place, offrant un cadre énonciatif propice à générer un certain nombre d'effets largement indépendants du contenu discursif proprement dit. En effet, c'est notre hypothèse que le succès de TLMEP dépend de sa mise en scène de certaines des tensions caractéristiques de la société québécoise contemporaine, notamment sur le plan identitaire, et que cette mise en scène dépend avant tout du dispositif particulier (et notamment technique) mis en place par l'émission.

\section{Les trois espaces}

Clarifions tout de suite la voie qui est la nôtre à l'intérieur du domaine très large de l'analyse des médias d'actualité, que Jean-Pierre Esquenazi résume en quatre perspectives distinctes : 1 . l'étude du rôle des médias ; 2 . la sociologie du journalisme ; 3 . l'étude de la réception ; 4 . l'analyse du discours (2002, p. 6). Si l'on excepte la première perspective, qui tend à en proposer une coupe transversale et qui relève d'une problématique toute différente de la nôtre, c'est un découpage en trois temps ou, mieux encore, en trois espaces que laisse deviner une telle cartographie de la recherche. En effet, tout produit médiatique implique trois espaces sociaux différents: celui de sa production (perspective 2 ), celui de sa réception (perspective 3 ) et enfin celui, moins facile à définir, qui est engendré par le caractère discursif de l'objet (perspective 4), et qu'Esquenazi, s'inspirant du modèle linguistique, nomme espace déictique en référence au " ici et maintenant " de la situation de communication, tels qu'ils s'incarnent dans un dispositif donné.

Traditionnellement, chacun de ces espaces a donné lieu à des analyses distinctes, largement autonomes, qui correspondent par ailleurs à des spécialisations dans les champs de la sociologie et de la sémiologie. Du côté de la production, par exemple, on peut penser aux travaux de pionnier de Gaye Tuchman (1978), à l'ouvrage d'Alain Accardo sur les Journalistes au quotidien ou encore à la réflexion de Pierre Bourdieu (1996) sur l'impact de la télévision sur le champ journalistique dans son ensemble; du côté de la réception, on se doit d'évoquer les études fondées sur un important travail statistique (Souchon, 1980), les travaux inspirés par la tradition des Uses and gratification (Katz, Blumler et Gurevitch,1973-1974), mais également ceux qu'on associe généralement à la mouvance des Cultural studies (Fiske, 1989); enfin, les analyses textuelles d'inspiration linguistique ou sémiologique sont de loin les plus nombreuses à s'être penchées sur les " objets » médiatiques eux-mêmes (journaux, émission, etc.) ; les travaux menés par Guy Lochard et Éric Soulages 
Le dispositif télévisuel de la version québécoise de Tout le monde en parle : espace déictique, identité discursive et cadre participatif

(1998), Noël Nel (1990) ou François Jost (2004), pour ne considérer que la France, semblent les plus représentatifs à cet égard.

Nous adoptons pour notre part un point de vue qui est nettement textuel ; fondée sur une observation minutieuse des dispositifs télévisuels, nous considérons comme primordial d'ancrer notre connaissance de la vie sociale des médias dans une conception des plus concrètes de l'objet comme processus symbolique. Toutefois, nous faisons nôtre la critique mainte fois adressée à la sémiologie et aux diverses méthodologies d'analyse de contenu de tenir peu compte du contexte particulier dans lequel sont élaborés les contenus médiatiques, et encore moins des caractéristiques des publics auxquels ils s'adressent. Nous croyons avec Esquenazi (2002) à l'importance de ne pas considérer l'élaboration des dispositifs médiatiques comme des textes à la signification enclose une fois pour toutes, mais comme des discours ouverts tout à la fois sur leur situation d'énonciation et sur leur public institutionnel.

Dans cette perspective, on ne cherche plus à saisir la signification univoque de ces discours (dont on peut d'ailleurs se demander si elle existe); mais à comprendre d'une part comment ils manifestent les étapes de leur production et d'autre part comment ils suscitent des interprétations, des appropriations ou des dénégations (p.10).

Autrement dit, le dispositif médiatique - ici une émission de télévision, mais ce pourrait être un journal, un film documentaire, etc., - peut avantageusement être lu, en amont, comme une paraphrase de l'institution qui lui donne vie et, en aval, comme une traduction des communautés interprétatives auxquelles il s'adresse. II s'agit en fait de saisir «l'objet » dans ce qu'il exprime d'une situation sociale, sans pour autant renoncer à tenir compte du caractère on ne peut plus tangible de la médiation qu'il opère, notamment à travers sa dimension technique. Appelons socio-sémiotique un tel cadre qui travaille à comprendre le travail des médias d'une façon qui rapproche plutôt qu'elle ne sépare la réalité du monde et ses mises en scène, sans pour autant tomber dans les pièges du miroir ou du reflet.

En accord avec notre conception de l'espace déictique, l'objectif est donc de rendre compte, une fois accomplie l'étape proprement descriptive, d'une part de la manière dont le dispositif manifeste l'" état du champ » en construisant son identité discursive, et d'autre part du type de cadre participatif qu'il induit. II est à noter qu'une telle stratégie méthodologique - inspirée en partie de l'écriture de l'actualité. Pour une sociologie du discours médiatique, de Jean-Pierre 
Pierre BARRETTE

Esquenazi (2007) - renvoie comme il se doit au cadre général de la sociosémiotique tout juste évoqué, dont les outils ne se limitent pas à l'analyse du discours de l'information, mais permettent également d'approcher des émissions de divertissement ou ce qu'il est convenu d'appeler des œuvres, comme le même auteur en fait la démonstration dans Sociologie des œuvres, de la production à l'interprétation.

\section{L'espace déictique de Tout le monde en parle}

Nous allons tenter dans les prochaines pages de décrire au mieux le dispositif télévisuel qui caractérise TLMEP, étant entendu pour nous que celui-ci est un important vecteur de production de sens. D'une certaine façon, on peut dire du dispositif qu'il précède, rend possible et encadre la prise de paroles des protagonistes de l'énonciation; il constitue un "cadre " à l'intérieur duquel sont régulés les jeux de langage et où sont produits des effets de mise en scène. Comme le fait très justement remarquer Patrick Amey : " dès lors que le signe se déprend de la contiguïté et de la contingence de son référent (la chose), et que se déploie l'intentionnalité d'un acteur agissant, affleure l'idée qu'un dispositif prend en charge du sens et de la forme, des énoncés et de l'énonciation » (Amey, 2009, p.17). Reste à en isoler les éléments ; Jean-Pierre Esquenazi suggère de considérer le dispositif médiatique en tant qu'« organisation de l'espace, organisation du temps et agencement d'énonciation " (Esquenazi, 1998, p. 115), une conception qui a le mérite de rendre compte de la totalité du phénomène et de son aspect continu, tout en permettant une souplesse qui évite de s'embrouiller de détails qui alourdiraient inutilement l'analyse. On notera que dans la mesure où il ne s'agit pas de catégories discrètes ni mutuellement exclusives, il n'est pas toujours aisé d'y assigner tel ou tel élément analysé.

Les observations qui suivent constituent la synthèse d'une écoute assidue, effectuée sur un corpus de 13 émissions, diffusées entre le 21 septembre 2011 et le 18 mars 2012. Dans la mesure où ce sont les agencements de base récurrents du dispositif qui nous intéressent, nous nous limitons ici à la description de ce qui constitue la " matrice » commune à toutes les occurrences

de TLMEP - ses invariants, si l'on préfère - en insistant tout particulièrement par ailleurs sur ce qui distingue ce dispositif de celui des talk-shows plus traditionnels. 
Le dispositif télévisuel de la version québécoise de Tout le monde en parle: espace déictique, identité discursive et cadre participatif

\subsection{Organisation spatiale}

- Le studio : pour l'enregistrement de TLMEP, le studio 42 de la tour de Radio-Canada prend nettement la forme d'une agora (voir l'annexe 1), dont les similitudes avec l'Assemblée nationale (le parlement québécois) sont particulièrement frappantes (annexes 2 et 3 ). De chaque côté du pupitre central d'où l'animateur Guy A. Lepage dirige les entrevues, deux minces tables où prennent place les invités se font face, formant des demi-cercles qui se prolongent vers le public en studio, lui-même distribué selon des cercles concentriques de plus en plus larges jusqu'à rejoindre, symboliquement, le public à la maison. On notera qu'une telle visibilité du public n'est pas si fréquente dans les talk-shows, si l'on excepte la catégorie des reality shows des années 1990, qui lui faisait une place de choix.

Mises à part ces similitudes, tout a été mis en œuvre pour évacuer le mieux possible la dimension austère des architectures parlementaires ; y dominent des couleurs chaudes (le bleu surtout, le vert et le rouge de façon moindre) dans un décor plutôt minimaliste et de facture contemporaine. En outre, il est absolument remarquable qu'une boule disco trône bien en vue au-dessus du décor, et toute une gamme d'effets visuels sophistiqués est rendue possible grâce à cet artefact ainsi qu'à un système d'éclairage performant. On notera par ailleurs que Guy A. Lepage dispose à la place qui lui est assignée d'une sorte de clavier qui lui permet d'avoir directement le contrôle de plusieurs des effets visuels et sonores en question.

- L'appareillage technique : habituellement invisible ou présenté le plus discrètement possible dans les talk-shows traditionnels, débats et autres émissions d'information (et a fortiori en fiction, où "montrer le dispositif » constitue la plupart du temps une erreur grossière), l'appareillage technique est ici tout à fait visible. Non seulement on ne déploie aucun effort pour dérober au regard du téléspectateur caméras et cameramen, moniteurs, rampe d'éclairage et "spots", mais il apparaît clairement que leur présence à l'écran est souhaitée et valorisée. La régisseuse de plateau fait de nombreuses apparitions à l'écran, munie de son casque d'écouteurs bien en vue, au point qu'elle est devenue une espèce de figure familière de l'émission, au même titre que l'animateur et son complice. L'espace du studio est ainsi présenté comme largement transparent; un espace de mise en scène, certes, mais qui donne comme « naturel » les outils de sa monstration.

- La nature des plans : cet espace scénique " unifié » n'est présenté par un plan d'ensemble qu'à de rares moments charnières de l'émission (essentiellement au début de l'émission et à l'occasion de l'entrée en scène d'un 
nouvel invité). Le reste du temps, l'espace du studio est morcelé par une large panoplie de plans (plan frontal, "yeux dans les yeux ", lorsque l'animateur s'adresse à la caméra; plan rapproché ou gros plan des invités lorsque ceux-ci répondent aux questions, etc.). Un type de plan en particulier est caractéristique de l'esthétique de l'émission et représente bien la conception de l'espace-temps sous-jacente : ce plan, qui présente au sein de l'image un moniteur où se trouve l'interviewé, on pourrait l'appeler " recadrage ", double écran ou encore " mise en abyme ". II est extrêmement fréquent tout au long de l'émission, au point de constituer une sorte de figure visuelle récurrente. II permet notamment de dynamiser l'image en intégrant une forme de montage dans le plan ; il renvoie en ce sens à une obsession propre à TLMEP, celle de contrer à tout prix le statisme et l'inertie souvent associés au genre du talk-show. Non seulement les plans sont-ils très courts ( 3 à 5 secondes en moyenne), mais ils doivent de surcroît assurer leur propre dynamisme interne.

\subsection{Organisation temporelle}

$-1^{\text {er }}$ niveau de découpage, les entrevues : on l'a mentionné un peu plus haut, le rythme de TLMEP est un aspect très important de son identité esthétique. Ce rythme est assuré, notamment, par un fractionnement de l'émission en blocs distincts, qui ont chacun leur couleur et leur unité. L'arrivée de chaque invité sur le plateau est soulignée par une musique qui accompagne son déplacement des coulisses vers la place qui lui est assignée, créant une césure temporelle entre les blocs. Cette musique est, la plupart du temps, liée au thème de l'entrevue sur un mode humoristique, distancié ou ironique. À mesure que l'émission avance, les invités sont appelés à rester autour de la table, faisant en sorte que le plateau est significativement plus populeux à la fin de l'émission qu'il ne l'est au début. Un autre marqueur temporel important prend place environ à la moitié du programme, lorsque Guy A. Lepage annonce que le moment est arrivé d'ouvrir une bouteille de vin ; l'émission entre alors en quelque sorte dans sa seconde phase, souvent plus festive et « relaxe » que la première.

$-2^{\mathrm{e}}$ niveau de découpage, les segments d'entrevue : si l'entrevue ellemême constitue l'unité de base du découpage de l'émission, la plupart des entrevues sont construites en plusieurs parties. Le modèle le plus fréquent suit un développement en trois temps : le premier segment situe le choix de l'invité par rapport à l'actualité de la semaine, le second retrace les principaux éléments de sa biographie et le troisième poursuit l'entrevue sur un mode plus ludique. II est à noter que seuls les invités ayant une notoriété assez importante et profitant d'un statut de personnalité publique ont droit aux segments 
Le dispositif télévisuel de la version québécoise de Tout le monde en parle: espace déictique, identité discursive et cadre participatif

biographiques et à la partie la plus ludique de l'entretien. Les autres, qui doivent habituellement leur présence à une visibilité ponctuelle dans l'actualité, ont droit à une entrevue dans laquelle sont surtout évoqués les motifs qui ont justifié l'invitation.

$-3^{\mathrm{e}}$ niveau de découpage : le montage : TLMEP est tourné en studio le jeudi soir, puis passe sous le couperet du monteur (Guy A. Lepage lui-même) le vendredi et le samedi avant d'être diffusée en heure de grande écoute le dimanche. Pour environ 6 à 7 heures de tournage, le produit final dure autour de 2 h 15 , ce qui implique un montage très serré des entrevues, d'où est éliminé tout ce qui est jugé par le monteur moins intéressant et moins pertinent. II arrive même que des entrevues complètes soient éliminées du montage final. Cette caractéristique, même si elle passe largement inaperçue pour une portion importante du public, est essentielle, car c'est grâce à ce rythme soutenu, et l'impression concomitante qu'un feu roulant de bons mots, de blagues et d'affirmations-chocs constitue l'ordinaire de ce plateau, que TMLEP arrive à trouver son identité particulière.

\subsection{Agencement d'énonciation}

- Rituel d'ouverture : sur le plan proprement énonciatif, le moment le plus important et significatif de l'émission est son ouverture. Guy A. Lepage y sort des coulisses, accompagné par la chanson thème du générique, il embrasse la régisseuse de plateau puis descend quelques marches, ce qui l'amène sur le plateau ; là, il entreprend de serrer la main de chaque membre du public qui se trouve à la première rangée puis il gagne sa chaise. Une fois à sa place, il regarde la caméra et présente, en adresse directe au public, les invités de la semaine qui prendront place sur le plateau durant les deux heures suivantes ; il annonce ensuite l'entrée sur le plateau du fou du roi, Dany Turcotte.

- Les postures énonciatives : TLMEP est l'affaire d'un animateur, d'un coanimateur et de leurs invités. Cette formule n'est certes pas neuve, puisque déjà dans les années 1970 le talk-show emblématique de la SRC (Appelez-moi Lise, que Lise Payette animait, assistée de Jacque Fauteux) y avait recours, parmi d'autres. La principale singularité de TLMEP réside toutefois dans le style particulier d'animation qu'on y adopte, un style favorisé par la personnalité des deux comparses qui se sont d'abord fait connaître en tant qu'humoristes. Guy A. Lepage, il nous semble, apporte avec lui une double persona: celle de leader du groupe Rock \& belles oreilles, connu pour ses charges tous azimuts dont plusieurs ont une importante coloration politique; celle de producteur, scénariste et acteur de la très populaire série Un gars, une fille dont le principal 
fait d'armes réside dans son succès international et la "résistance » de son idéateur aux producteurs étrangers (notamment américains) qui désiraient l'adapter sans respect pour l'intégrité artistique de la série.

Danny Turcotte, le Fou du roi, ancien membre du Groupe sanguin, y contribue pour sa part en amenant sur le plateau un sens de la répartie cinglante ainsi qu'un humour souvent cynique ; mais c'est son homosexualité, révélée au grand public sur le plateau même de l'émission dès le début de la seconde saison, qui sert le mieux sa position dans l'émission en lui permettant d'asseoir fortement le rôle de " clown blanc » d'emblée dévolu au personnage, et ce dans les deux versions (française et québécoise) de TLMEP.

Cette animation à deux têtes imprime un style particulier aux entrevues, notamment en " triangulant » le face à face énonciatif. Les questions que pose Lepage sont nettement "scriptées », presque mises en scène, puisqu'il les lit précautionneusement, souvent maladroitement à partir d'un carton, un peu comme si quelqu'un d'autre que lui-même les avait écrites. Les questions et interventions de Dany Turcotte sont au contraire "spontanées » et lancées à brûle-pourpoint ; du moins sont-elles présentées telles.

- Les invités, axe paradigmatique : les invités semblent être choisis en fonction d'une logique qui est d'abord paradigmatique : sur une année, on peut estimer que l'ensemble des domaines de l'actualité courante sont à peu près couverts (politique provinciale, fédérale et municipale, économie, showbiz, sport, société, littérature, etc.). Bien sûr, les aléas de l'actualité ont une incidence importante sur la composition du plateau (par exemple, durant les élections, les chefs des différents partis sont invités à l'émission à tour de rôle), mais une catégorie d'invités en particulier semble échapper plus que les autres à ces impondérables : les humoristes. Très peu d'éditions de TLMEP, en effet, se sont passées complètement de la présence d'un de ces représentants du monde du spectacle, et il est remarquable qu'ils sont largement majoritaires sur la courte liste des détenteurs d'une " carte chouchou ", cette dernière donnant le droit à l'invité qui en est le détenteur de revenir quand bon lui semble sur le plateau de l'émission.

- Invité, axe syntagmatique : il est clair, par ailleurs, que la liste d'invités est conçue chaque semaine dans le but de respecter un certain équilibre et de favoriser des échanges entre participants, bref d'assurer une bonne " chimie " sur le plateau. On comprend mieux dans ce contexte que, au-delà des efforts réels pour échantillonner l'actualité de manière minimalement représentative, le critère qui l'emporte est souvent celui de la notoriété des personnalités invitées et de leur capacité à donner un "bon spectacle », d'où la place de choix systématiquement offerte aux humoristes. 
Le dispositif télévisuel de la version québécoise de Tout le monde en parle : espace déictique, identité discursive et cadre participatif

\section{Identité discursive et cadre participatif}

Cette synthèse de nos observations, on l'aura compris, n'a pas la prétention d'être exhaustive, mais elle permet croyons-nous d'établir l'essentiel de ce qui constitue le dispositif particulier de TLMEP, en deçà du contenu conversationnel qu'il encadre. Nous allons maintenant tenter de comprendre comment ces éléments du dispositif télévisuel, déployés en heure de grande écoute à RadioCanada, contribuent d'une part à l'identité discursive de la Société d'État, et d'autre part proposent un cadre de participation au public de la chaîne. En effet, " toute institution médiatique est d'abord partie prenante du champ social dont elle relève " (Esquenazi, 1998, p. 129), ce qui la force à se positionner, à prendre parti face à la concurrence, autrement dit à se doter d'une identité discursive ; par ailleurs, cette institution, à travers ses programmes, revendique " une forme de communication », elle propose au public un cadre participatif auquel ce dernier est libre ou non de se plier. Ces deux "faces" du programme, l'une qui regarde en amont vers son contexte de production, l'autre en aval vers les conditions de sa réception, constituent pour nous la meilleure manière de rendre compte de sa dimension sociale.

Tentons d'abord de situer rapidement TLMEP à l'intérieur des limites de l'infodivertissement. Par rapport à d'autres émissions québécoises participant du phénomène - pour rester à la SRC, pensons à Infoman ou encore à Et Dieu créa Laflaque ${ }^{1}$ - TLMEP propose un contenu à la fois moins politique (un invité par semaine, la plupart du temps) et moins ouvertement parodique. Laflaque, par exemple, qui calque son format sur celui d'un bulletin de nouvelles, fait la part belle aux personnalités les plus en vue de la politique à tous les niveaux de gouvernement, et les personnages récurrents de l'émission (les Marois, Charest, Labeaume, Tremblay) appartiennent presque tous au champ de la politique partisane. Même chose du côté d'Infoman, dont l'ordre du jour est très près de celui d'un journaliste traditionnel, même si le rendu, lui, reste très différent. Malgré ces différences, les trois émissions forment ensemble une sorte de petite constellation radio-canadienne au cœur de laquelle se joue un traitement des affaires publiques marqué par une légèreté de ton que ne connaît pas le téléjournal, principal véhicule de l'information politique " sérieuse »; s'y cristallise donc d'une façon qui semble largement solidaire la version québécoise du phénomène de l'infodivertissement.

1 Infoman est une émission de télévision québécoise d'une demi-heure présentant l'actualité politique, culturelle et sociale de façon satirique ; Et Dieu créa... Laflaque est un show sarcastique de marionnettes qui commente l'actualité par le biais de son personnage principal, Gérard D. Laflaque, père de famille et annonceur de nouvelles. 
TLMEP est possiblement l'émission dont le dispositif rend compte avec le plus de transparence des antagonismes qui caractérisent le champ médiatique où elle prend place. La disposition scénique, qui rappelle comme on l'a fait remarquer l'architecture de l'assemblée nationale, impose l'image d'une agora, d'un lieu sacré où la parole est reine et transcendante; le choix d'invités politiques et de défenseurs de causes diverses, la déférence appuyée avec laquelle on accueille ces derniers, la présence silencieuse d'un public à l'écoute et les airs contrits que se donne à l'occasion l'animateur tirent l'émission du côté des affaires publiques et renvoient directement au mandat de télévision publique de Radio-Canada (en gros : informer, cultiver, distraire). Mais dans un même temps, toute une série de traits propres au dispositif fait basculer l'émission du côté du pur divertissement : la boule disco, d'abord, à laquelle on a conféré le pouvoir de transformer la sérieuse agora en discothèque festive; la présence systématique (à commencer par l'équipe d'animation) sur le plateau d'humoristes patentés, de loin la catégorie la mieux représentée parmi la liste générale des invités, et quasiment la seule à figurer sur celle des chouchous ; la nature même des entrevues, qui fait régulièrement une place importante à la parodie, à la distanciation, à la dérision; le service du vin à mi-parcours, qui contribue à rendre festive l'atmosphère sur le plateau ; le montage extrêmement serré de l'émission, qui sacrifie toute séquence qui ne se conforme pas à un modèle très précis d'efficacité dramatique; etc. Face à un tel dédoublement d'identité, on peut difficilement ne pas penser au "conflit d'allégeances » qui sévit à l'intérieur même des murs de la société d'État et considérer le dispositif de TLMEP comme une réponse aux divergences idéologiques concernant le rôle dévolu à la télévision publique dans l'espace social (Tremblay et Moeglin, 2005), ou comme le propose Bourdieu (1996), comme un effort pour se distinguer de ses concurrents sans cesser de se conformer à la logique du champ.

Cet aspect de la composition d'une identité discursive par l'émission vedette de la SRC concerne aussi la situation de compétition vécue à la télévision québécoise le dimanche soir. Depuis plusieurs années, le réseau TVA et RadioCanada mènent une lutte sans merci dans ce créneau horaire, que la principale chaîne privée a choisi pour la diffusion de certains de ses plus gros canons, dont Le Banquier (version québécoise de Deal or no deal), Star Académie, On connaît la chanson (version québécoise de Don't Forget the Lyrics), Occupation double et - même s'il n'a pas connu le succès escompté Québec-Montréal (téléréalité mettant en scène une compétition entre deux équipes de hockey amateur). Toutes ces émissions ont en commun de jouer à divers titres la carte de la téléréalité, un "genre » dans lequel sont mis de l'avant des quidams, des Monsieur-et-Madame-tout-le-monde auxquels la télévision promet les « quinze minutes de gloire » jadis prophétisées par Andy 
Le dispositif télévisuel de la version québécoise de Tout le monde en parle: espace déictique, identité discursive et cadre participatif

Warhol. La télévision généraliste publique se trouve dans une position ambiguë et souvent malaisée de compétition avec cette télévision privée, qui a découvert dans les formats de téléréalité une réponse à ses difficultés.

Contre ces "blockbusters " télévisuels, organisés autour de dispositifs qui mettent en scène la transformation d'anonymes en célébrités instantanées (Jost, 2009), Radio-Canada a choisi d'opposer une conception beaucoup plus institutionnelle de la célébrité. En effet, c'est un peu comme si le succès de TLMEP tenait d'abord... au succès des gens qu'on y invite. Entendons, cela va de soi, une construction délibérée de l'idée de succès, qui dépend ici très largement d'un culte de la personnalité, partout visible dans les médias, mais qui prend sur le plateau de Guy A. Lepage la forme aiguë d'un motif récurrent et exclusif, qui a par ailleurs partie prenante avec le statut de cet animateur: acteur, réalisateur, producteur, animateur, ce parangon médiatique en est venu à symboliser dans l'esprit de bien des Québécois non seulement une des rares réussites internationales de la télévision québécoise - son émission Un gars une fille est un grand succès un peu partout dans le monde - , mais également la Résistance: il tient tête aux Américains, à qui il refuse de laisser carte blanche pour l'adaptation de son concept. Le dispositif de l'émission, qui fait sienne la persona d'animateur de Lepage, contribue donc à nourrir l'identité discursive de la chaîne publique en affirmant son indépendance vis-à-vis des formats les plus en vogue (la téléréalité), et en particulier par rapport au discours populiste véhiculé par ce genre.

Ceci ne veut pas dire pour autant que le téléspectateur n'est pas sollicité par le dispositif de TLMEP. Plusieurs éléments contribuent en effet à suggérer un cadre participatif fortement inclusif, lisible telle une sorte "d'appel » à la constitution d'un espace de convivialité " ouvert » et " distinctif ». La séquence d'ouverture de l'émission est à ce titre assez remarquable : l'insistance que met l'animateur à faire littéralement le tour du studio pour serrer la main de chacun des membres de la première rangée est à entendre comme une stratégie de contact qui travaille à ritualiser formellement le rapport au public et ainsi à favoriser son inclusion dans le dispositif d'énonciation. II nous semble par ailleurs que les efforts affectés à la monstration du dispositif (écran dans l'écran, apparition dans le champ des cameramen et de la régisseuse de plateau, présence des rails d'éclairage, etc.) vont dans le même sens, suggérant un espace dans lequel la technologie est "transparente ", sans faux-fuyant, un espace qualifié par un régime de visibilité maximale. L'homosexualité déclarée du coanimateur, qui a comme on le sait choisi de faire son coming out médiatique sur le plateau de l'émission, et l'image qui en résulte d'une différence à la fois assumée et intégrée, contribuent aussi - tout comme les diverses causes à connotations identitaires que lui et Lepage endossent 
publiquement dans le cadre de l'émission - à faire du plateau de TLMEP un excellent exemple de la mouvance postmoderne, dans laquelle les revendications identitaires particularistes prennent parfois le pas sur un projet politique rassembleur et unifié.

L'utilisation par Guy A. Lepage depuis maintenant quelques mois du réseau social Twitter pour suivre et commenter en direct - l'enregistrement se fait le jeudi soir - le déroulement de sa propre émission contribue à cette idée de forum élargi, et montre bien comment l'interactivité peut désormais s'appliquer à des contextes communicationnels pour lesquels elle n'avait pas d'abord été pensée. II y a quelque chose d'un peu surréaliste et d'assez troublant en effet que d'assister à une émission de TLMEP alors que fusent les commentaires, le public - une toute petite partie, certes, mais qui est appelée à grossir - se constituant tout à coup en une sorte de "communauté seconde " qui donne corps pour une rare fois à l'idée de public de la télévision, une entité éminemment flottante qui se définit au mieux statistiquement. Le contexte domestique dans lequel on regarde la télévision engage au contraire du cinéma une pratique éminemment privée qui tend à évacuer la dimension communautaire. La mise à profit de Twitter concourt en quelque sorte à placer la diffusion de l'émission en réseau et en temps réel, et par le fait même à rapprocher l'expérience télévisuelle d'une sorte de happening participatif dont la dimension sociale est incontestable.

\section{Conclusion}

Au terme de notre parcours, la "posture " sociale de Tout le monde en parle devrait se trouver éclairée. Son dispositif particulier, qui joue d'un habile tricotage entre les affaires publiques et le divertissement sans pour autant appartenir d'emblée à l'un ou l'autre des genres, apparaît telle une paraphrase de l'institution où il prend place: s'y révèle la nature fortement clivée, hétéronome d'un champ où s'exercent des pressions à la fois symboliques et économiques. Le recours systématique à l'humour n'y est pas aussi innocent qu'on voudrait le croire. Comme le mentionne très justement Gilles Lipovetsky (1984) : "Quand le social entre dans la phase humoristique, commence le néonarcissisme, dernier refuge cérémoniel d'un monde sans puissance supérieure. À la dévalorisation parodique du social répond le sur-investissement liturgique du Moi. »

II y a bien de cela ici : le défilé des ego surdimensionnés " dont tout le monde parle " ne fait pas une société ; il arrive même très mal à en proposer une image. D'où, en aval, l'impérieux dessein placé au cœur même du dispositif 
Le dispositif télévisuel de la version québécoise de Tout le monde en parle:

espace déictique, identité discursive et cadre participatif

d'offrir un cadre de participation au téléspectateur qui soit comme une recomposition de l'espace communautaire, et de s'adjoindre pour ce faire les derniers moyens technologiques (Twitter, comme on l'a vu, mais aussi Facebook) qui semblent redonner au destinataire les rènes de la communication en le plaçant lui aussi en position de prise de parole. La télévision de masse, la télévision rassembleuse peut difficilement aujourd'hui faire l'économie d'une interactivité de principe. La dimension proprement politique de TLMEP ne se situe donc pas tant pour nous dans la présence régulière sur le plateau de l'émission des figures les plus connues de la politique partisane; elle relève bien davantage de la constitution au travers d'un dispositif donné d'un simulacre d'espace public qui s'offre telle une réponse aux ratés de la politique institutionnelle.

\section{Bibliographie}

ACCARDO A., 1995, Journalistes au quotidien, Bordeaux, Le Mascaret.

AMEY P., 2009, La parole à la télévision. Les dispositifs des talk-shows, Paris, L'Harmattan.

BARRETTE P., PAQUETTE É, et PERRATON C (Dir), Dérives de l'espace public à l'ère du divertissement, Montréal, PUQ.

BASTIEN, F., 2007, Entre la peur et la confiance: I'histoire, le contenu et l'auditoire des émissions d'information politique et d'infodivertissement à la télévision francophone au Québec, Thèse de doctorat en science politique, Université de Montréal.

BLOUIN M.-C., 2007, Tout le monde en parle, un miroir révélateur de la société québécoise ?, Mémoire de maîtrise, UQAM.

BOUGNOUX D., 1995, La Communication contre l'information?, Paris, Hachette.

BOURDIEU P., 1996, Sur la télévision, Paris, Liber.

BRANTS K., 2003, « De l'art de rendre la politique populaire... Ou qui a peur de l'infotainment ? », Réseaux, 2003/2, n 118, pp. 135-166.

ESQUENAZI J.-P., 2002, L'écriture de l'actualité. Pour une sociologie du discours médiatique, Grenoble, PUG. 
ESQUENAZI J.-P., 2007, Sociologie des æuvres, de la production à l'interprétation, Paris, Armand Colin.

FISKE J., 1989, Television Culture, Londres, Routledge.

JOST F., 2004, Introduction à l'analyse de la télévision, Paris, Ellipses.

JOST F., 2004, Grandeur et misères de la téléréalité, Paris, Le cavalier bleu.

LE FOULGOC, A., 2003, « 1990-2002 : une décennie de politique à la télévision française. Du politique au divertissement », Réseaux, 2003/2, n 118, pp. 2363.

LIPOVETSKY D., 1984, L'ère du vide, Paris : Gallimard.

LOCHARD G. et SOULAGES J.-C., 1998, La communication télévisuelle, Paris, A. Collin.

KATZ E., BLUMLER, G. et GUREVITCH M. (1973-1974), "Uses and Gratifications Research », The Public Opinion Quarterly, vol. 37, n 4, pp. 509-23.

NEL N., 1990, Le débat télévisé, Paris. A. Collin.

NEVEU É., 2003, «De l'art (et du coût) d'éviter la politique. La démocratie du talk-show version française (Ardisson, Drucker, Fogiel) », Réseaux, 2003/2, $\mathrm{n}^{\circ} 118$, pp. 95-134.

PELISSIER N., 2001 Les mutations du journalisme à l'heure des nouveaux réseaux numériques, Annuaire Français des Relations Internationales, pp. 912-930.

ROCHETTE É., 2010, Le comportement communicationnel des politiciens à Tout le monde en parle, Mémoire de maîtrise, Université Laval.

SOUCHON M., 1980, Petit écran Grand public, Paris, La documentation française.

THUSSU, D. K., 2007, News as Entertainment: The Rise of Global Infotainment, Thousand Oaks, Calif. : Sage.

TREMBLAY G. et Moeglin P., 2005, L'avenir de la télévision généraliste, Paris, L'Harmattan.

TUCHMAN G., 1978, Making News, A Study in the construction of reality, New York, The Free Press. 
Le dispositif télévisuel de la version québécoise de Tout le monde en parle : espace déictique, identité discursive et cadre participatif

\section{Annexe 1 : Schéma du plateau de TLMEP}


Pierre BARRETTE

\section{Annexe 2 : Le plateau de TLMEP}

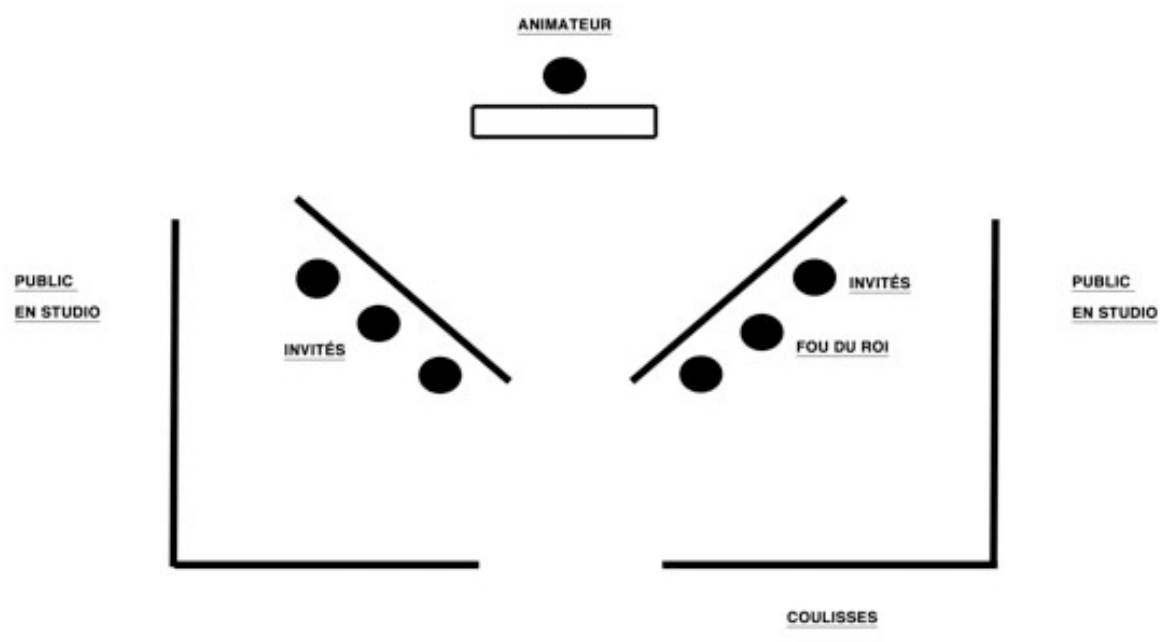


Le dispositif télévisuel de la version québécoise de Tout le monde en parle : espace déictique, identité discursive et cadre participatif

\section{Annexe 3 : L’Assemblée nationale du Québec}

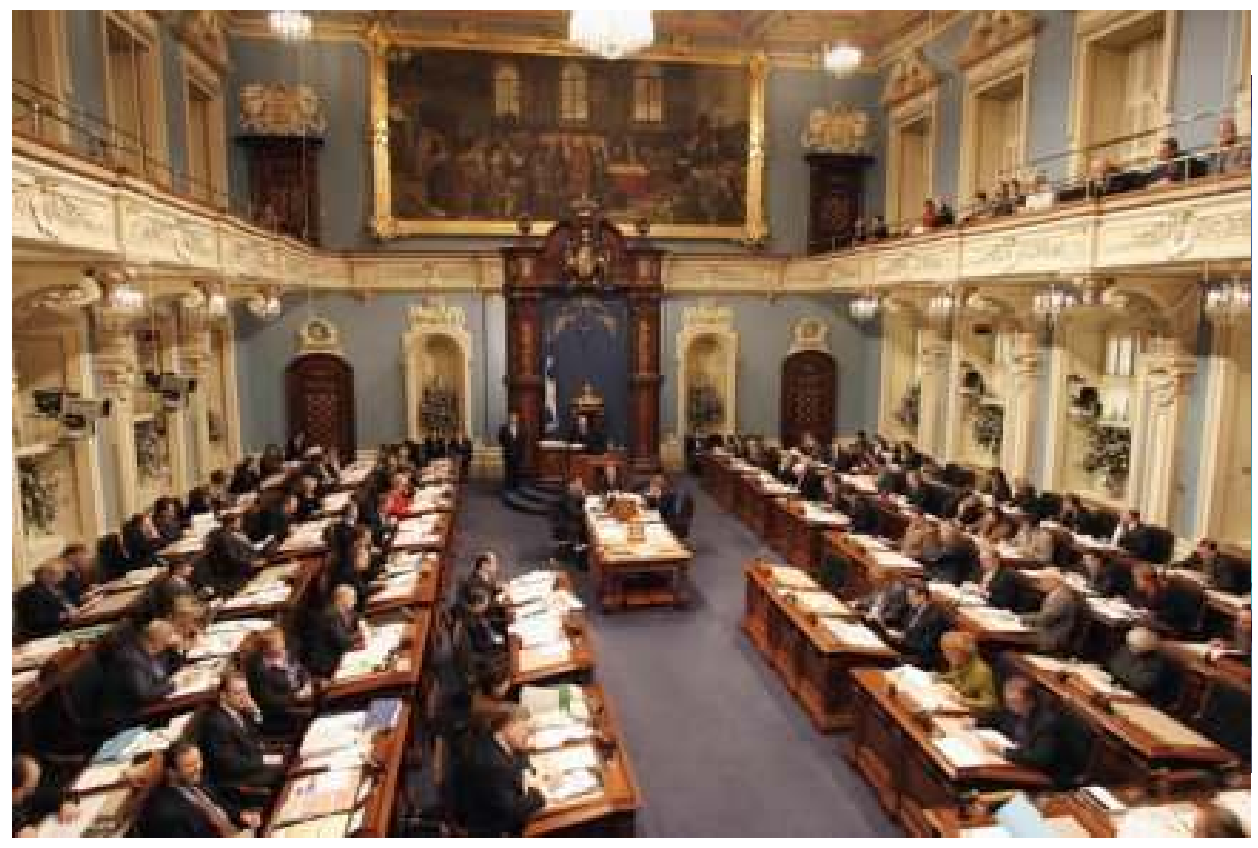

